\title{
NUMBER OF MINIMAL COMPONENTS AND HOMOLOGICALLY INDEPENDENT COMPACT LEAVES FOR A MORSE FORM FOLIATION
}

\author{
IRINA GELBUKH* \\ Department of Mathematics, Moscow State University, Russia \\ Current address: CIC, IPN, 07738, DF, Mexico \\ e-mail: gelbukh@member.ams.org \\ Communicated by A. Némethi
}

(Received May 3, 2007; accepted May 5, 2008)

\begin{abstract}
The numbers $m(\omega)$ of minimal components and $c(\omega)$ of homologically independent compact leaves of the foliation of a Morse form $\omega$ on a connected smooth closed oriented manifold $M$ are studied in terms of the first non-commutative Betti number $b_{1}^{\prime}(M)$. A sharp estimate $0 \leqq m(\omega)+c(\omega) \leqq b_{1}^{\prime}(M)$ is given. It is shown that all values of $m(\omega)+c(\omega)$, and in some cases all combinations of $m(\omega)$ and $c(\omega)$ with this condition, are reached on a given $M$. The corresponding issues are also studied in the classes of generic forms and compactifiable foliations.
\end{abstract}

\section{Introduction and announce of the results}

Consider a connected closed oriented manifold $M$ with a Morse form $\omega$, i.e., a closed 1-form with Morse singularities $\operatorname{Sing} \omega$ (locally the differential of a Morse function). This form defines a foliation $\mathcal{F}_{\omega}$ on $M \backslash \operatorname{Sing} \omega$.

The number $m(\omega)$ of minimal components and $c(\omega)$ of homologically independent compact leaves are important topological characteristics of the foliation. For example, if $\mathcal{F}_{\omega}$ is compactifiable, i.e. $m(\omega)=0$, then $\operatorname{rk} \omega \leqq c(\omega)$, where $\mathrm{rk} \omega$ is the number of its incommensurable periods; for the the cycle rank $m(\Gamma)$ of the foliation graph $\Gamma$ it holds $m(\Gamma)=c(\omega)$ (Section 2.1; [4]).

Considerable effort has been devoted to estimating these numbers. Obviously, $c(\omega) \leqq b_{1}(M)$, where $b_{1}(M)$ is the Betti number; in [1] (dim $\left.M \geqq 3\right)$ and [7] $\left(M_{g}^{2}\right)$ it was shown that $2 m(\omega) \leqq b_{1}(M)$. In [4] these facts were

2000 Mathematics Subject Classification. Primary 57R30, 58K65.

Key words and phrases. Morse form foliation, minimal components, compact leaves.

0081-6906/\$ 20.00 (c) 2009 Akadémiai Kiadó, Budapest 
combined into

$$
0 \leqq c(\omega)+2 m(\omega) \leqq b_{1}(M)
$$

In [11] it was shown that $c(\omega) \leqq h(M)$, where $h(M) \leqq b_{1}(M)$ is another homological characteristic of the manifold; in [4] this was generalized to an independent estimate

$$
0 \leqq c(\omega)+m(\omega) \leqq h(M)
$$

An independent estimate in terms of Sing $\omega$ was given in [12]:

$$
0 \leqq c(\omega)+m(\omega) \leqq \frac{\left|\Omega_{1}\right|-\left|\Omega_{0}\right|}{2}+1,
$$

where $\Omega_{1}$ is the set of conic singularities and $\Omega_{0}$ of centers. These estimates were, though, not exact.

In this paper we give an exact estimate in terms of the non-commutative Betti number $b_{1}^{\prime}(M)$ - the maximal rank of a free quotient group of the fundamental group $\pi_{1}(M)$ [9]; obviously $b_{1}^{\prime}(M) \leqq b_{1}(M)$ and as we show, $b_{1}^{\prime}(M) \leqq h(M)$. We prove (Theorem 3) that

$$
0 \leqq c(\omega)+m(\omega) \leqq b_{1}^{\prime}(M)
$$

and show that all intermediate values are reached on $M$ even for $c(\omega)$ alone:

$$
0 \leqq c(\omega) \leqq b_{1}^{\prime}(M)
$$

and even in the class of compactifiable foliations (Theorem 8). In particular, on any $M$ there exists a compactifiable foliations with all (compact) leaves being homologically trivial; such forms are exact (Theorem 4). On $M_{g}^{2}$, all combinations of $c(\omega)$ and $m(\omega)$ that satisfy (2) are reached (Propositon 7); $b_{1}^{\prime}\left(M_{g}^{2}\right)=g$ (Lemma 2). Possibly all combinations of $c(\omega)$ and $m(\omega)$ that satisfy both (1) and (2) are reached on a given manifold (Conjecture 11); these conditions are independent if $\operatorname{dim} M \geqq 3$ (Remark 9, Example 10).

A Morse form is called generic if each its singular leaf contains a unique singularity [2]; such forms are dense in the space of Morse forms. All statements mentioned above hold in the class of generic forms, with some exceptions for $M_{g}^{2}$ (Remark 12). Specifically, the exact lower bound in (2) on $M_{g}^{2}$ except for $S^{2}$ is 1 (Proposition 14):

$$
1 \leqq c(\omega)+m(\omega) \leqq b_{1}^{\prime}\left(M_{g}^{2}\right)=g
$$


and for compactifiable foliations of generic forms on $M_{g}^{2},(3)$ is reduced to $c(\omega)=g$ (Lemma 13, Remark 16). With this, for generic forms on $M_{g}^{2}$ possible are all combinations of $c(\omega)$ and $m(\omega)$ such that if $\mathcal{F}_{\omega}$ is compactifiable then $c(\omega)=g$, otherwise $1 \leqq c(\omega)+m(\omega) \leqq g$ (Proposition 17).

The paper is organized as follows. In Section 2 we give necessary definitions and prove some useful facts. In Section 3 we prove the main inequality (2). In Section 4 we prove the exactness of this inequality by constructing forms with extremal values. In Section 5 we show that all intermediate values within the bounds (2) are reached, and in some cases all values of $c(\omega)$ and/or $m(\omega)$ allowed by (2) are reached (this does not eliminate the simpler Section 4 since its examples are used as building blocks). Finally, in Section 6 we give analogs of our most important statements for the class of generic forms.

\section{Definitions and useful facts}

In this paper, $M$ is a connected closed oriented manifold. A closed 1-form $\omega$ on $M$ is called a Morse form if it is locally the differential of a Morse function. The set Sing $\omega=\{p \in M \mid \omega(p)=0\}$ of its singularities is finite, since they are isolated and $M$ is compact. In this paper we consider only singular forms, i.e., Sing $\omega \neq \emptyset$. On $M \backslash \operatorname{Sing} \omega$ the form $\omega$ defines a foliation $\mathcal{F}_{\omega}$.

\subsection{Leaves and components; $c(\omega)$ and $m(\omega)$}

A leaf $\gamma \in \mathcal{F}_{\omega}$ is called compactifiable if $\gamma \cup \operatorname{Sing} \omega$ is compact; otherwise it is called non-compactifiable. A foliation is called compactifiable if all its leaves are compactifiable. The number of non-compact compactifiable leaves $\gamma_{i}$ is finite, since each singularity can compactify no more than four leaves.

A singular leaf $\gamma^{0}$ is a maximal union of one or more leaves and one or more singularities such that for any two points $p, q \in \gamma^{0}$ there exists a path $\alpha:[0,1] \rightarrow M$ with $\alpha(0)=p, \alpha(1)=q$ and $\omega(\dot{\alpha}(t))=0$ for all $t$.

A Morse form (or function) is called generic if each its singular leaf contains a unique singularity [2]. Generic forms are dense in the space of Morse forms.

By $m(\omega)$ we denote the number of minimal components of $\mathcal{F}_{\omega}$. A minimal component is a connected component of the union of non-compactifiable leaves. The latter union is open, the number of minimal components is finite, and each non-compactifiable leaf is dense in its minimal component $[1,6]$. Obviously, $\mathcal{F}_{\omega}$ is compactifiable if $m(\omega)=0$.

Lemma 1. On $M_{g}^{2}$, a minimal component contains two cycles $z, z^{\prime}$ such that $z \cdot z^{\prime} \neq 0$. 
Proof. Let $U$ be a minimal component and $s \subset U$ a curve such that $\int_{s} \omega \neq 0$. Consider the cycle in $H_{1}(\bar{U}, \partial \bar{U})$ corresponding to $[s] \in H_{1}(U)$. By Poincaré duality it defines a non-zero cocycle $\alpha \in H^{1}(U, \mathbb{Z})$. Since torsion $\left(H_{1}\left(M_{g}^{2}\right)\right)=0,[s]$ can be viewed as an element of Hom $\left(H_{1}(U), \mathbb{Z}\right)$, i.e. $\alpha(z)=[s] \cdot z$. Since $\alpha \neq 0$ there exists $z \in H_{1}(U)$ such that $[s] \cdot z \neq 0$.

By $c(\omega)$ we denote the number of homologically independent compact leaves of $\mathcal{F}_{\omega}$. For a compact leaf $\gamma$ there exists an open neighborhood consisting solely of compact leaves: indeed, integrating $\omega$ gives a function $f$ with $d f=\omega$ near $\gamma$; hence the union of all compact leaves is open.

A connected component of the union of compact leaves of $\mathcal{F}_{\omega}$ is called a maximal component. Since Sing $\omega \neq \emptyset$, it is a (maximal) cylindrical neighborhood $\gamma \times(0,1)$ of any its leaf $\gamma \in \mathcal{F}_{\omega}$ and consists of compact leaves diffeomorphic to $\gamma$. Its boundary is a union of some non-compact compactifiable leaves and singularities. Obviously, the number of maximal components is finite [4].

The foliation graph $\Gamma$ is the graph whose edges are maximal components (their boundary has one or two connected components) and vertices are connected components of the union of all non-compact leaves, i.e., a vertex consists of singularities, singular leaves, and/or minimal components; an edge is incident to a vertice if they adjoin in $M$. The structure of the foliation graph closely reflects that of the foliation itself; see details in [4]. In particular,

$$
m(\Gamma)=c(\omega)
$$

where $m(\Gamma)$ is the cycle rank [5] of the graph.

By rk $\omega$ we denote the number of incommensurable periods of the form $\omega$, i.e., $\operatorname{rk} \omega=\operatorname{rk}_{\mathbb{Q}}\left\{\int_{z_{1}} \omega, \ldots, \int_{z_{k}} \omega\right\}$, where $z_{1}, \ldots, z_{k}$ is a basis of $H_{1}(M)$. If $\mathcal{F}_{\omega}$ is compactifiable then

$$
\operatorname{rk} \omega \leqq c(\omega)
$$

in particular, $c(\omega)=m(\omega)=0$ implies $\omega=d f[4]$.

\subsection{Non-commutative Betti number $b_{1}^{\prime}(M)$}

By $b_{1}^{\prime}(M)$ we denote the non-commutative Betti number - the maximal rank (number of free generators) of a free quotient group of $\pi_{1}(M)$ [1]; $b_{1}^{\prime}(M) \leqq b_{1}(M)$, the Betti number [9].

LEMMA 2. $b_{1}^{\prime}\left(M_{g}^{2}\right)=g$. 

group

Proof. Let $M=M_{g}^{2}$. Obviously, $b_{1}^{\prime}(M) \geqq g$ since the fundamental

$$
\pi_{1}\left(M_{g}^{2}\right)=\left\langle a_{i}, b_{i}, i=1, \ldots, g \mid a_{1} b_{1} a_{1}^{-1} b_{1}^{-1} \ldots a_{g} b_{g} a_{g}^{-1} b_{g}^{-1}=1\right\rangle
$$

can be mapped onto a free subgroup $\left\langle a_{i}, i=1, \ldots, g\right\rangle$. Let us show $b_{1}^{\prime}(M) \leqq g$.

Given a surjection $\pi_{1}(M) \rightarrow F, \operatorname{rk} F=b_{1}^{\prime}(M)$, consider a continuous map $f: M \rightarrow W, W=\bigvee_{i=1}^{b_{1}^{\prime}(M)} S_{i}^{1}$. Let $p_{i} \in S_{i}^{1}$ be its regular values; $c_{i}=f^{-1}\left(p_{i}\right)$ are circles in $M$. Consider the map $f_{*}: H_{1}(M) \rightarrow H_{1}(W)$. Cycles $z_{i} \in$ $H_{1}(M)$ such that $f_{*} z_{i}=\left[S_{i}^{1}\right] \in H_{1}(W)$ are independent. By construction $\left[c_{i}\right] \cdot z_{j}=\delta_{i j}$, therefore $\left[c_{i}\right], i=1, \ldots, b_{1}^{\prime}(M)$, are also independent in $H_{1}(M)$. Since $\left[c_{i}\right] \cdot\left[c_{j}\right]=0$, we obtain $b_{1}^{\prime}(M) \leqq g$.

A Morse form (or a minimal component) is called weakly complete if it has no centers and any its singular leaf containing a conic singularity (of index 1 or $n-1$ ) stays connected after removal this singularity. In any nonzero cohomology class there exists a weakly complete Morse form [8].

\section{Main theorem: bounds on $c(\omega)+m(\omega)$}

Theorem 3. Let $M$ be a smooth closed oriented manifold and $\omega$ a Morse form on it. Then

$$
0 \leqq c(\omega)+m(\omega) \leqq b_{1}^{\prime}(M)
$$

and all intermediate values are reached on a given $M$; in particular, the bounds are exact.

Proof. (i) $\operatorname{dim} M \geqq 3$. Let $\mathcal{F}_{\omega}$ contain $m_{1}$ not weakly complete and $m_{2}$ weakly complete minimal components, $m_{1}+m_{2}=m(\omega)$. By [9, Theorem I.1] the fundamental group of the space of leaves $\pi_{1}(M / \omega)$ can be represented as a free product of free abelian groups

$$
\pi_{1}(M / \omega)=(\underbrace{\mathbb{Z} * \cdots * \mathbb{Z}}_{k_{0}}) *(\underbrace{\mathbb{Z} * \cdots * \mathbb{Z}}_{k_{1}}) *\left(P_{1} * \cdots * P_{m_{2}}\right),
$$

where the first $k_{0}$ factors correspond to the set of the compact leaves and form $\pi_{1}(\Gamma)$ ( $\Gamma$ is the foliation graph); the next $k_{1}$ factors correspond to the set of weakly complete minimal components, $k_{1} \geqq m_{1}$; and the groups $P_{i}$ correspond to weakly complete minimal components, rk $P_{i} \geqq 2$, with $k_{0}+$ $k_{1}+m_{2} \leqq b_{1}^{\prime}(M)$.

Since $k_{0}=m(\Gamma)$, the latter inequality and (4) implies (6).

Erratum:

$\mathrm{k} 1$ factors correspond to the set of not weakly complete minimal components 
(ii) $\operatorname{dim} M=2$. Let $\gamma_{1}, \ldots, \gamma_{c}, c=c(\omega)$, be homologically independent compact leaves and $U_{1}, \ldots, U_{m}, m=m(\omega)$, minimal components of $\mathcal{F}_{\omega}$. By Lemma 1 there exist $z_{i}, z_{i}^{\prime} \subset U_{i}$ such that $z_{i} \cdot z_{i}^{\prime} \neq 0$. The cycles $\left[\gamma_{1}\right], \ldots,\left[\gamma_{c}\right]$, $z_{1}, \ldots, z_{m}$ are independent; indeed,

$$
\left(\sum_{i=1}^{c} n_{i}\left[\gamma_{i}\right]+\sum_{i=1}^{m} m_{i} z_{i}\right) \cdot z_{j}^{\prime}=0
$$

implies all $n_{i}, m_{i}=0$. Moreover, all $\left[\gamma_{i}\right] \cdot\left[\gamma_{j}\right]=\left[\gamma_{i}\right] \cdot z_{j}=z_{i} \cdot z_{j}=0$. Thus $c+m \leqq g=b_{1}^{\prime}\left(M_{g}^{2}\right)$ (by Lemma 2 ).

Existence of all values within the bounds (6) follows from Theorem 8 below. Exactness of the bounds also independently follows from Theorem 4 and Proposition 5.

\section{Existence of extremal values of $c(\omega)$ and $m(\omega)$}

Theorem 4. On $M$ there exists a Morse form $\omega$ with $c(\omega)=m(\omega)=$ 0 , i.e., $\mathcal{F}_{\omega}$ being compactifiable and all its leaves homologically trivial (such forms are exact).

Proof. Exactness of the form follows from (5). We will construct a Morse function $f$ with $c(d f)+m(d f)=0$.

(i) $\operatorname{dim} M \geqq 3$. Consider a tubular neighborhood $Y$ of a wedge sum $\bigvee_{i=1}^{b_{1}(M)} S_{i}^{1}$ of circles that generate a basis of $H_{1}(M) ; \partial Y$ is connected and homologically trivial. Let $\partial Y$ be a leaf of $f$.

The inside of $Y$ can be foliated as shown in Fig. 1. The figure shows the neighborhood of a wedge sum of (two) circles $S_{i}^{1}$ (the edges of the cylinders are identified). Take a center $p_{0}$; surrounding leaves are spheres. Extend them along $S_{1}^{1}$ until they self-intersect forming a conic singularity $p_{1}$ and then an $S^{1} \times S^{n-2}$. Extend the latter along $S_{2}^{1}$ until it self-intersects forming a conic singularity $p_{2}$. Repeating this for all $S_{i}^{1}$ will foliate $Y$ such that all leaves are homologically trivial and $\partial Y$ is a leaf.

Now extend $f$ on the rest of $M$; all its leaves are homologically trivial. Indeed, denote $M^{\prime}=\overline{M \backslash Y} ; \partial M^{\prime}=\partial Y$. By construction, $H_{1}\left(M^{\prime}, \partial M^{\prime}\right)=$ 0 , then

$$
H^{n-1}\left(M^{\prime}, \mathbb{Z}\right)=H_{n-1}\left(M^{\prime}\right) \oplus \text { torsion }\left(H_{n-2}\left(M^{\prime}\right)\right)=0
$$

by the Poincaré duality. We obtain $H_{n-1}\left(M^{\prime}\right)=0$.

(ii) $\operatorname{dim} M=2$. On $S^{2}$ all leaves are homologically trivial. Let $M=M_{g}^{2}$, $g \geqq 1$. Fig. $2(a)$ shows a torus $T^{2}$ (the opposite sides of the square are 


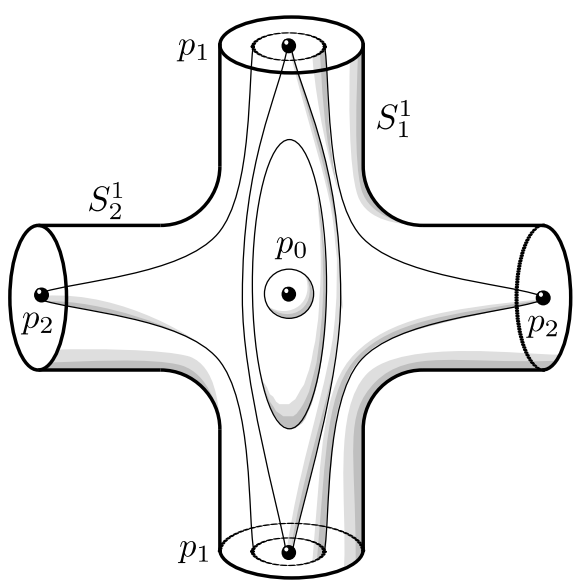

Fig. 1. Foliating the inside of $Y$

identified) with a desired foliation: $p_{i}$ are centers and $q_{i}$ saddles. Finally, $M_{g}^{2}=\sharp_{i=1}^{g} T_{i}^{2}$ is assembled as a connected sum of tori, see Fig. $2(b)$ : a leaf surrounding $p_{2}$ of each previous torus is identified with a leaf surrounding $p_{1}$ of the next torus.

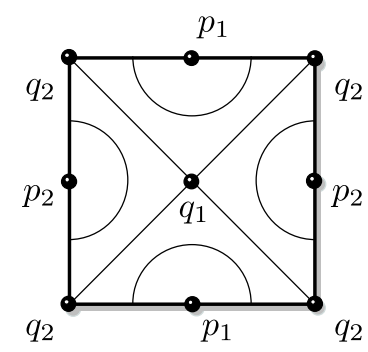

$(a)$

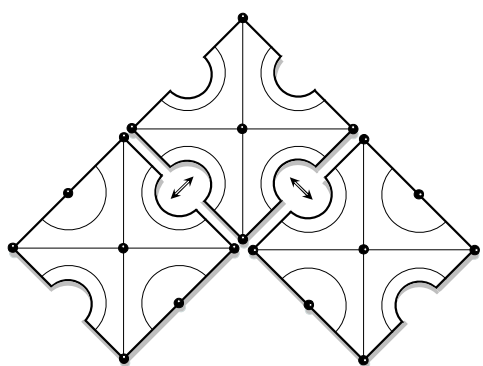

(b)

Fig. 2. Compactifiable foliation with $c(\omega)=0$ on $(a) T^{2}$, (b) $M_{g}^{2}=\sharp T_{i}^{2}$

Proposition 5. On $M$ there exists a Morse form $\omega$ with $c(\omega)=b_{1}^{\prime}(M)$ and $m(\omega)=0\left(\mathcal{F}_{\omega}\right.$ compactifiable $)$.

Proof. By definition of $b_{1}^{\prime}(M)$ there exists a surjective homomorphism $\pi_{1}(M) \rightarrow F$, where $F$ is a free group, $r k F=b_{1}^{\prime}(M)$. Consider a corresponding map $\varphi: M \rightarrow W$, where $W=\bigvee_{i=1}^{b_{1}^{\prime}(M)} S_{i}^{1}$. Let $\alpha_{W} \in H^{1}(W, \mathbb{R})$, $\operatorname{rk} \alpha_{W}=b_{1}^{\prime}(M)$, and $\alpha=\varphi^{*} \alpha_{W}$.

Let $x_{i} \in S_{i}^{1}$ be regular values of $\varphi$; each $M_{i}=\varphi^{-1}\left(x_{i}\right)$ is a compact submanifold of $M$. Denote by $M^{\prime}$ the result of cutting $M$ open along the $M_{i}$; 
$\partial M^{\prime}=\bigcup_{i}\left(M_{i}^{+} \cup M_{i}^{-}\right)$. We obtain $\left.\alpha\right|_{M^{\prime}}=0$. Thus we can choose on $M^{\prime}$ a Morse function $f$ without singularities on $\partial M^{\prime}$ such that it is constant on each connected component of $\partial M^{\prime}, f\left(M_{i}^{+}\right)-f\left(M_{i}^{-}\right)=\int_{S_{i}^{1}} \alpha$, and $\left.f\right|_{\partial M^{\prime}}$ fits together smoothly, giving on $M$ a Morse form $\omega \sim \alpha$. Obviously, $\mathcal{F}_{\omega}$ is compactifiable; thus by (5) it holds $c(\omega) \geqq \operatorname{rk} \omega=b_{1}^{\prime}(M)$. From Theorem 3 it follows $c(\omega)=b_{1}^{\prime}(M)$ and $m(\omega)=0$.

Proposition 6. If $b_{1}(M) \geqq 2$ then on $M$ there exists a Morse form $\omega$ with minimal foliation; in particular, $c(\omega)=0$ and $m(\omega)=1$.

Proof. For $\operatorname{dim} M \geqq 3$ this was proved in [1]. A corresponding foliation on $M_{g}^{2}=\sharp T_{i}^{2}$ is shown in Fig. 3 .

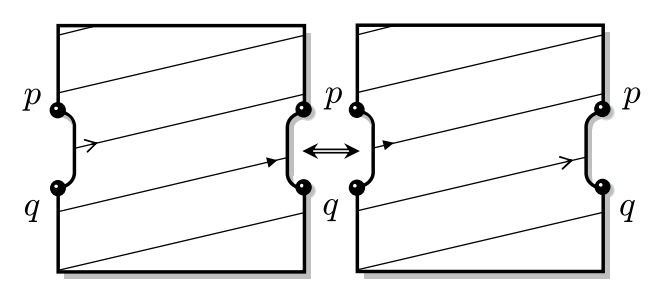

Fig. 3. Minimal foliation on $M_{g}^{2}=\sharp\left(T_{i}^{2}\right)$

5. Existence of intermediate values of $c(\omega)$ and $m(\omega)$

Proposition 7. Let $c, m \in \mathbb{Z}$. On $M_{g}^{2}$ there exists a Morse form $\omega$ such that $c(\omega)=c$ and $m(\omega)=m$ iff

$$
0 \leqq c+m \leqq b_{1}^{\prime}\left(M_{g}^{2}\right)=g
$$

Proof. By Theorem 3 and Lemma 2, we only need to show existence. To construct the desired $\omega$ represent $M_{g}^{2}$ as a connected sum of $c$ tori with a compact, and $m$ with a minimal, non-singular foliation plus an $M_{g-c-m}^{2}$ foliated as in Theorem 4, glued together by a circle inserted between leaves via a saddle as shown in Fig. 4. iff

Theorem 8. Let $c \in \mathbb{Z}$. On $M$ there exists a Morse form $\omega$ with $c(\omega)=c$

$$
0 \leqq c \leqq b_{1}^{\prime}(M)
$$

The form can be chosen with $m(\omega)=0\left(\mathcal{F}_{\omega}\right.$ compactifiable). 


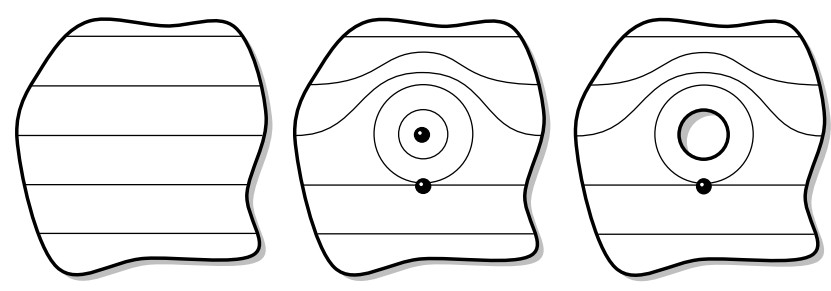

Fig. 4. Preparing a summand for the connected sum

Proof. By Theorem 3 we only need to show existence. For $\operatorname{dim} M=2$ see Proposition 7 ; let $\operatorname{dim} M \geqq 3$. By Proposition 5 , on $M$ there exists a Morse form $\omega_{0}$ with compactifiable foliation and $c\left(\omega_{0}\right)=b_{1}^{\prime}(M)$. Starting from this foliation, we will construct a compactifiable foliation with $c(\omega)=c$.

Let $\gamma_{1}, \ldots, \gamma_{c}$ be homologically independent compact leaves of $\mathcal{F}_{\omega_{0}}$. Denote by $\mathcal{M}$ the result of cutting $M$ open along $\gamma_{i} ; \partial \mathcal{M}=\bigcup_{i}\left(\gamma_{i}^{+} \cup \gamma_{i}^{-}\right)$. We will construct on $\mathcal{M}$ a form $\omega$ with no homologically non-trivial leaves other than connected components of $\partial \mathcal{M}$, which are $\gamma_{i}$. We have done this for $\mathcal{M}=M(c=0, \partial \mathcal{M}=\emptyset)$ in Theorem 4 .

As in that theorem, consider a tubular neighborhood $Y$ of a wedge sum $\bigvee_{i} S_{i}^{1}$ of circles that generate a basis of $H_{1}(\mathcal{M})$, foliate it as shown in Fig. 1, and extend the obtained Morse function $f$ to the rest of $\mathcal{M}$. We need, however, a closer look at $\mathcal{M}^{\prime}=\overline{\mathcal{M} \backslash Y}$ than (7), since now $\partial \mathcal{M}^{\prime}=\partial Y \cup \partial \mathcal{M}$.

By construction, $i_{*} H_{1}\left(\partial \mathcal{M}^{\prime}\right)=H_{1}\left(\mathcal{M}^{\prime}\right)$, where $i: \partial \mathcal{M}^{\prime} \rightarrow \mathcal{M}^{\prime}$ is the inclusion map. Let us consider the commutative diagram:

$$
\begin{array}{ccc}
H_{n-1}\left(\mathcal{M}^{\prime}, \partial \mathcal{M}^{\prime}\right) & \stackrel{\partial}{\rightarrow} & H_{n-2}\left(\partial \mathcal{M}^{\prime}\right) \\
\downarrow & & \downarrow \\
H^{1}\left(\mathcal{M}^{\prime}\right) & \stackrel{i^{*}}{\rightarrow} & H^{1}\left(\partial \mathcal{M}^{\prime}\right)
\end{array}
$$

where vertical arrows are Poincaré duality. Since by construction $i_{*}$ is surjective, we have $\operatorname{ker} i^{*}=0$. Thus $\operatorname{ker} \partial=0$. Consider the long exact sequence of a pair:

$$
\rightarrow H_{n-1}\left(\partial \mathcal{M}^{\prime}\right) \stackrel{i_{*}}{\rightarrow} H_{n-1}\left(\mathcal{M}^{\prime}\right) \stackrel{j}{\rightarrow} H_{n-1}\left(\mathcal{M}^{\prime}, \partial \mathcal{M}^{\prime}\right) \stackrel{\partial}{\rightarrow} H_{n-2}\left(\partial \mathcal{M}^{\prime}\right) \rightarrow
$$

Since $\operatorname{im} j=\operatorname{ker} \partial=0$, we obtain $H_{n-1}\left(\mathcal{M}^{\prime}\right)=i_{*} H_{n-1}\left(\partial \mathcal{M}^{\prime}\right)$. Thus leaves of $f$ on $\mathcal{M}$ are homologous to 0 or $\gamma_{i}$.

Again, we may assume that $\left.f\right|_{\partial \mathcal{M}}$ fits together smoothly, giving on $M$ a Morse form $\omega$ with $c(\omega)=c$. Obviously, the corresponding foliation is compactifiable. 
REMARK 9. If $\operatorname{dim} M \geqq 3$, not all combinations of $c(\omega)$ and $m(\omega)$ allowed by Theorem 3 may be possible. Inequality (1) imposes additional restrictions on $m(\omega)$ if $b_{1}^{\prime}(M)>\frac{1}{2} b_{1}(M)$. The latter values are independent: for a torus $T^{n}$ it holds $b_{1}^{\prime}\left(T^{n}\right)=1, b_{1}\left(T^{n}\right)=n$; for a connected sum $M=\sharp_{i=1}^{m}\left(S^{n-1} \times\right.$ $\left.S^{1}\right)$ it holds $b_{1}^{\prime}(M)=b_{1}(M)=m[1]$.

Example 10. Let $M=S^{2} \times S^{1}$; obviously, $b_{1}^{\prime}(M)=b_{1}(M)=1$. Though Theorem 3 allows $m(\omega)=1$, (1) prohibits it.

Conjecture 11. On $M$ there exist Morse forms with all combinations of $c(\omega)$ and $m(\omega)$ that satisfy (6) and (1).

\section{Generic forms}

REMARK 12. Theorems 3, 4, and 8 hold in the class of generic forms for $\operatorname{dim} M \geqq 3$. Propositions 5 and 6 hold in this class for any $M$.

Indeed, the corresponding Morse forms or functions constructed in their proofs are generic. However, for $M_{g}^{2}$ the three theorems, as well as Proposition 7 , should be modified to hold in the class of generic forms.

For the following fact proved in [10], we give a shorter independent proof.

Lemma 13 (see [10]). On $M_{g}^{2}$, if $\omega$ is generic and $m(\omega)=0\left(\mathcal{F}_{\omega}\right.$ compactifiable) then $c(\omega)=g$.

Proof. Consider the foliation graph $\Gamma$. Its cycle rank $m(\Gamma)=N_{e}-N_{v}+$ 1 , where $N_{e}$ is the number of edges and $N_{v}$ of vertices [5]. Since $\omega$ is generic and $\mathcal{F}_{\omega}$ compactifiable, vertices of $\Gamma$ are of indices 1 or $3: 2 N_{e}=n_{1}+3 n_{3}$, where $n_{i}$ is the number of vertices of index $i$ [5]. So $2 m(\Gamma)=n_{3}-n_{1}+2$. Obviously, $n_{1}=\left|\Omega_{0}\right|$ and $n_{3}=\left|\Omega_{1}\right|$, where $\Omega_{0}$ is the set of centers and $\Omega_{1}$ of conic singularities. By (4), we have $2 c(\omega)=\left|\Omega_{1}\right|-\left|\Omega_{0}\right|+2$. On the other hand, on $M_{g}^{2}$ it holds $\left|\Omega_{1}\right|-\left|\Omega_{0}\right|=2 g-2$. We obtain $c(\omega)=g$.

Proposition 14. The statement of Theorem 3 holds for generic forms except that on $M_{g}^{2}, g \geqq 1$, the exact lower boundary in (6) is 1 :

$$
1 \leqq c(\omega)+m(\omega) \leqq b_{1}^{\prime}\left(M_{g}^{2}\right)=g
$$

Proof. That 0 in (6) is unreachable for a generic form on $M_{g}^{2}, g \neq 0$, follows from Lemma 13, which together with Lemma 2 gives

$$
c(\omega)+m(\omega)=c(\omega)=b_{1}^{\prime}\left(M_{g}^{2}\right)=g .
$$


Existence of all intermediate values in (6) in the class of generic forms follows from Proposition 17 and Theorem 8 (Remark 12). Exactness of the lower bound also independently follows from Proposition 15 and Proposition 6 and that of the upper bound from Proposition 5 (Remark 12).

Proposition 15. The statement of Theorem 4 holds for generic forms iff $\operatorname{dim} M \geqq 3$ or $M=S^{2}$.

Proof. For exclusion of $M_{g}^{2}, g \geqq 1$, see Lemma 13 .

REMARK 16. Similarly, the statement of Theorem 8 holds for generic forms except that on $M_{g}^{2}$ the form cannot be chosen with $m(\omega)=0$ unless $c(\omega)=g$.

Proposition 17. Let $c, m \in \mathbb{Z}$. On $M_{g}^{2}$ there exists a generic Morse form $\omega$ such that $c(\omega)=c$ and $m(\omega)=m$ iff either $m>0$ and $1 \leqq c+m \leqq g$ or $m=0$ and $c=g$ (cf. Proposition 7).

Proof. By Lemma 13 and Proposition 14, we only need to show existence. If $m=0$, represent $M_{g}^{2}$ as a connected sum of $g$ tori with a compact non-singular foliation. Otherwise, represent it as a connected sum of $c$ tori with a compact, and $m-1$ with a minimal, non-singular foliation plus an $M_{g-c-m+1}^{2}$ with a foliation as in Proposition 6 (Remark 12).

\section{REFERENCES}

[1] Arnoux, P. and Levitt, G., Sur l'unique ergodicité des 1-formes fermées singulières, Invent. Math., 84 (1986), no. 1, 141-156. MR 87g:58004

[2] Farber, M., Topology of closed one-forms, Math. Surv. and Monographs, AMS, v. $108,2004 . M R$ 2005c: 58023

[3] Gelbukn, I., Presence of minimal components in a Morse form foliation, Diff. Geom. Appl., 22 (2005), no. 2, 189-198. MR 2005 m:57040

[4] Gelbukn, I., On the structure of a Morse form foliation, Czechoslovak Mathematical Journal, 59 (2009), no. 1, 207-220.

[5] Harary, F., Graph theory, Addison-Wesley Publ. Comp., 1994. MR 41\#1566

[6] Imanishi, H., On codimension one foliations defined by closed one forms with singularities, J. Math. Kyoto Univ., 19 (1979), no. 2, 285-291. MR 80k:57050

[7] Katok, A., Invariant measures for flows on oriented surfaces, Sov. Math., Dokl., 14 (1973), no. 3, 1104-1108. $M R \mathbf{4 8} \# 9771$

[8] Levitt, G., 1-formes fermées singulières et groupe fondamental, Invent. Math., 88 (1987), 635-667. MR 88d:58004

[9] Levitt, G., Groupe fondamental de l'espace des feuilles dans les feuilletages sans holonomie, J. Diff. Geom., 31 (1990), 711-761. MR 91d:57018

[10] Mel’nikova, I., An indicator of the noncompactness of a foliation on $M_{g}^{2}$, Math. Notes, 53:3 (1993), 356-358. MR 94h:57044

[11] Mel'nikova, I., A test for non-compactness of the foliation of a Morse form, Russ. Math. Surveys, 50:2 (1995) 444-445. MR 96f:57028

[12] Mel'nikova, I., Non-compact leaves of a Morse form foliation, Math. Notes, 63:6 (1998), 760-763. MR 2000e:57046 